\title{
Time-dependent modelling of PKS 2155-304 in a low state
}

\author{
M. Petropoulou ${ }^{\star}$
}

\author{
Department of Physics, Purdue University, 525 Northwestern Avenue, West Lafayette, IN, 47907, USA \\ e-mail: maroulaaki@gmail.com
}

Received 15 July 2014 / Accepted 29 August 2014

\begin{abstract}
Aims. We apply both leptonic and leptohadronic emission scenarios for modelling the multiwavelength photon spectra and the observed variability in the optical, X-ray, and TeV gamma-ray energy bands of blazar PKS 2155-304 while being in a low state between 25 August and 6 September 2008.

Methods. We consider three emission models, namely a one-component synchrotron self-Compton model (1-SSC), a one-zone proton synchrotron model (LHs), and a two-component SSC model (2-SSC). Only in the first scenario can the emission from the optical up to very high-energy (VHE) gamma-rays be attributed to a single particle population from one emission region. In the LHs model, the low-energy and high-energy bumps of the spectral energy distribution (SED) are the result of electron and proton synchrotron radiation, respectively, i.e. two different particle populations are required. In the 2-SSC model, the emission from one component dominates in the optical and gamma-ray energy bands, while the other one contributes only to the X-ray flux. Using a time-dependent numerical code that solves the kinetic equations for each particle species, we derived, in all cases, acceptable fits to the time-averaged SED. By imposing variations to one (or more) model parameters according to observed variability pattern in one (or more) frequencies we calculated the respective lightcurves and compared them with the observations.

Results. We show that the 1-SSC model cannot account for the anticorrelation observed between the X-rays and VHE gamma-rays, although it can explain the time-averaged SED. The anticorrelation can be more naturally explained by the two-component emission models. Both of them reproduce satisfactorily the optical, X-ray, and $\mathrm{TeV}$ variability but at the cost of additional free parameters, which from four in the 2-SSC model increase to six in the LHs model. Although the results of our time-resolved analysis do not favour one of the aforementioned models, they suggest that a two-component scenario is more adequate for the emission of PKS 2155-304 in the low state of 2008, which agrees with a recent independent analysis. This suggests that the quiescent blazar radiation might result from a superposition of the radiation from different components, while a flare might still be the result of a single component.
\end{abstract}

Key words. radiation mechanisms: non-thermal - gamma rays: galaxies - BL Lacertae objects: individual: PKS 2155-304 galaxies: active

\section{Introduction}

Blazars are a subclass of active galactic nuclei with a non-thermal emission covering most of the electromagnetic spectrum, i.e. from radio up to very high-energy (VHE) gammarays, and the dominant class of extragalactic sources at energies $>100 \mathrm{MeV}$ (Hinton \& Hofmann 2009; Holder 2012). Their broadband emission, which originates from a relativistic jet oriented close to our line of sight, is Doppler boosted and so it shows no evidence of spectral lines, at least for the subclass of BL Lac objects. The spectral energy distribution (SED) of TeV-emitting blazars consists of two smooth, broad components (e.g. Ulrich et al. 1997; Fossati et al. 1998). The first one extends from the radio up to the X-rays with a peak between the optical and soft X-ray energy bands, while the second one extends up to $\mathrm{TeV}$ energies, with a peak energy around $0.1 \mathrm{TeV}$, although this is not always clear (Abdo et al. 2011).

An important tool in our attempt to understand the physics of blazar emission is the modelling of their SED. Although it is a common belief that the lower energy bump is the synchrotron emission of relativistic electrons, the origin of the high-energy component is still under debate. Theoretical models are divided into leptonic and leptohadronic, according to the type of particles responsible for the gamma-ray emission. In leptonic scenar-

\footnotetext{
* NASA Einstein Postdoctoral Fellow.
}

ios, the high-energy component is the result of inverse Compton scattering of electrons in a photon field. As seed photons can serve the synchrotron photons produced by the same electron population (SSC models; e.g. Maraschi et al. 1992; Konopelko et al. 2003) or/and photons from an external region (EC models), for example from the accretion disk (Dermer et al. 1992; Dermer \& Schlickeiser 1993) or from the broad line region (Sikora et al. 1994; Ghisellini \& Madau 1996; Böttcher \& Dermer 1998). In leptohadronic scenarios, on the other hand, the gamma-ray emission can be the result of (i) proton synchrotron radiation (Mannheim \& Biermann 1992; Aharonian 2000; Mücke et al. 2003); (ii) synchrotron radiation of secondary pairs produced in the hadronic cascade (e.g. Petropoulou \& Mastichiadis 2012a; Mastichiadis et al. 2013); or even of (iii) neutral pion decay (Sahu et al. 2013; Cao \& Wang 2014). For a recent review on leptohadronic modelling, see Boettcher (2012) and Böttcher et al. (2013).

Stationary one-zone models have been extensively used and, in most cases, both leptonic and hadronic models prove equally successfull in fitting the SED of blazars (Maraschi et al. 1992; Sikora et al. 1994; Bloom \& Marscher 1996; Tavecchio et al. 1998; Cerruti et al. 2012; Reimer 2012; Böttcher et al. 2013; Dimitrakoudis et al. 2014; Cao \& Wang 2014). However, the recently obtained wealth of (quasi)simultaneous observations that cover both the low-and high-energy regimes of the SED, gives 
an opportunity for time-dependent modelling. Since leptonic and hadronic models are expected to have different time signatures, this time-resolved fitting analysis can pose new challenges to both categories of models. It can be used either to explain the origin of a flare (see e.g. Krawczynski et al. 2002 and references therein) or to lift the degeneracy between models that can successfully fit the time-averaged SED (Mastichiadis et al. 2013), yet, time-dependent blazar modelling has not been widely applied to quiescent emission mainly because of the lack of contemporeneous MW data in low states.

In the present work we aim to test whether one-zone emission models can account for both the observed SED and lightcurves of a blazar source, even when this is in a low state where the variability is marginal. We investigate, in particular, three possible scenarios in a time-dependent way: (i) a onezone SSC model, where the emission from the optical up to $\mathrm{TeV}$ gamma-rays is explained in terms of a relativistic electron distribution; (ii) a one-zone leptohadronic model (LHs), where the low-energy and high-energy humps are the result of electron and proton synchrotron radiation, respectively; and (iii) a two-component SSC model, where we assume that there are two physically distinct regions that contribute to the overall SED.

We apply our models to the multiwavelength observations of the high-peaked blazar (HBL) PKS 2155-304 at redshift $z=0.116$ in a low state (Aharonian et al. 2009b, henceforth, A09). The particular choice of data was motivated by the following: (i) the blazar was for the first time monitored simultaneously in four energy bands, namely in optical with ATOM (Hauser et al. 2004), in X-rays with RXTE (Jahoda et al. 1996) and Swift (Burrows et al. 2005), in GeV gamma-rays with Fermi (Atwood et al. 2009) and in $\mathrm{TeV}$ gamma-rays with H.E.S.S. (A09); (ii) it was observed in a low state with marginal variability at least at two energy bands (optical and GeV gammarays) implying that the underlying physical conditions do not vary significantly; (iii) a significant correlation between the optical and VHE gamma-ray fluxes was found, which is not commonly observed in HBLs (Krawczynski et al. 2004; Wagner 2008; Aharonian et al. 2009a) - see, however, Donnarumma et al. (2009) for a possible correlation observed during a flare of Mrk 421; and (iv) no correlation between the X-rays and VHE gamma-rays was detected in contrast to flaring events (Aharonian et al. 2009a; H.E.S.S. Collaboration et al. 2012). As we show in Sect. 4, it is the last two observational facts, in particular, that will be used to distinguish between the one- and two-component emission models.

For each one of the models, we find first a set of parameters that lead the system to a steady-state that fits in a broad sense the time-averaged SED. This is used as an initial condition for studying the properties of the source in the period MJD 54704-54715. For this, we vary one or more model parameters according to the temporal pattern observed in one or more energy bands and try to reproduce the observed variability in as many as possible energy bands. Although first results can be found in Petropoulou \& Mastichiadis (2013), here we extend the previous analysis by discussing in more detail the emission models and by presenting fiducial flaring episodes in the context of the 2-SSC model. We note also that Barres de Almeida et al. (2014) used the same dataset as an application of their method, which is based on studying the properties of optical polarization from blazar sources (Barres de Almeida et al. 2010), and, interestingly, the authors reached to similar conclusions to ours.

The present work is structured as follows: in Sect. 2 we describe the general framework and present in more detail our method in Sect. 3. We continue with the presentation of our results in Sect. 4 and conclude in Sect. 5 with a summary and discussion. Throughout this study we use $H_{0}=$ $70 \mathrm{~km} \mathrm{Mpc}^{-1} \mathrm{~s}^{-1}, \Omega_{\mathrm{M}}=0.3$ and $\Omega_{\Lambda}=0.7$.

\section{The model}

In what follows we use the one-zone leptohadronic model as described in (Dimitrakoudis et al. 2012, henceforth DMPR12) and for completeness we repeat here its basic points. We consider a spherical blob of fixed radius $R$ moving with a Doppler factor $\delta$ with respect to us and containing a magnetic field of strength $B$. We also assume that relativistic electrons and/or protons are being injected in the blob with a rate given by

$Q_{i}(\gamma, \tau)=Q_{0} \gamma^{-s_{i}} \mathrm{e}^{-\left(\frac{\gamma}{\gamma_{i, \max }}\right)^{q_{i}}} H\left(\gamma-\gamma_{i, \min }\right) H(\tau)$

where the subscript $i=p, e$ accounts for protons and electrons, $s_{i}$ is the power-law index, $H(x)$ is the Heaviside function, $\tau$ is the comoving time measured in units of the crossing time $t_{\mathrm{cr}}=R / \mathrm{c}$, and $Q_{0}$ is the amplitude of the injection rate. By setting $Q_{\mathrm{p}}=0$ the leptohadronic model simplifies into the usual SSC model ${ }^{1}$. The minimum and maximum Lorentz factors of the distribution are denoted as $\gamma_{i, \min }$ and $\gamma_{i, \max }$, respectively. The exponent $q_{i}$ determines the curvature of the exponential cutoff and typical values predicted by acceleration models are 1-2 (see e.g. Lefa et al. 2011). Finally, particles are also allowed to leave the region after a characteristic timescale $t_{i, \mathrm{esc}}=t_{\mathrm{cr}}$.

The injection rate can be associated with a compactness ${ }^{2}$ as

$\ell_{i}^{\text {inj }}=\frac{3 L_{i}^{\text {inj }} \sigma_{\mathrm{T}}}{4 \pi R m_{i} c^{3}}$,

where the injected luminosity in protons or electrons as measured in the comoving frame is given by

$L_{i}^{\mathrm{inj}}=V_{b} m_{i} c^{2} t_{\mathrm{cr}}^{-1} \int_{\gamma_{i, \text { min }}}^{\infty} \mathrm{d} \gamma \gamma Q_{i}(\gamma, \tau)$,

where the factor $t_{\mathrm{cr}}$ is introduced because the injection rate defined in Eq. (1) is expressed in terms of the dimensionless time $\tau$.

Protons can lose energy via three channels: (i) synchrotron radiation; (ii) photopair production (Bethe-Heitler); and (iii) photopion production. The relative effect of these three processes on the proton distribution function depends on the specific parameters of the system, therefore all three have to be taken into account in a kinetic equation, which besides the proton injection term, contains a proton escape term. Leptohadronic modelling is far more complex compared to SSC modelling mainly because of the creation of secondary particles, such as pions, which eventually decay to electron/positron pairs. Thus, one has to also follow the evolution of photons and electrons, by writing two additional kinetic equations for them that are being coupled to the equation of protons. Neutrons and neutrinos are also byproducts of photopionic interactions and, in principle, one has to include two more equations for them - see DMPR12. In this study, where the dominant energy loss process for protons is synchrotron radiation, one can safely ignore them.

At the cost of no spatial information, i.e. by assuming uniform distributions inside the blob, one can obtain a detailed picture of the particle distribution evolution in time and in energy

\footnotetext{
1 As there is no evidence of a strong external photon field in the environment of blazar PKS 2155-304, we use the terms "leptonic" and "SSC" models interchangeably.

2 Expression (2) contains a factor of three not present in the conventional definitions (see e.g. Petropoulou \& Mastichiadis 2011).
} 
by solving a system of coupled partial integrodifferential equations, which for leptohadronic models consists at least from five equations. However, in the case of SSC models, the system simplifies into a set of two equations, one for photons and one for electron/positron pairs.

The above scheme can be used to derive both steady-state and time-dependent solutions. If all parameters are constant in time the system will eventually reach a steady-state ${ }^{3}$. If, on the other hand, we allow for one or more parameters to have some explicit time dependence, such as $Q_{0}$ and $\gamma_{i, \max }$ in Eq. (1), then the system will not reach a steady state but it will show temporal variations, which will be associated with the variations imposed on the input parameters.

\section{The method}

The method we follow is similar to that described in Krawczynski et al. (2002) in that we attempt a time-dependent modelling of the observed lightcurves in one or more energy bands. Instead of approximating the time-variability by a sequence of steady states, we calculate the evolution of the electron distribution and the resulting photon spectra at each crossing time, which serves as a unit for the timestep used in the numerical code (for more details, see Mastichiadis \& Kirk 1995 and DMPR12). Moreover, the present work extends the previous analysis of Krawczynski et al. (2002) by attempting timedependent modelling of MW observations in the context of a leptohadronic model. It is noteworthy that for PKS 2155-304, in particular, only stationary solutions for its MW spectra were obtained within the leptohadronic context (Cerruti et al. 2012).

In order to model the MW data of PKS 2155-304 during the period MJD 54704-54715, we have considered the following three models listed in order of increasing complexity: (i) one-component SSC model (1-SSC); (ii) leptohadronic proton synchrotron model (LHs); and (iii) two-component SSC model (2-SSC). In all cases we have employed a four-step process:

1. We determine a set of parameters constant in time that leads the system to a steady state that lies close to but below the time-averaged SED.

2. We use this steady state as an initial condition of the system.

3. We impose variations to one or more parameters following the variability pattern observed in one or more energy bands.

4. We calculate the lightcurves for different values of the parameters that are related to the imposed variations. In case we do not find an acceptable fit to at least one of the observed lightcurves we go to step (1) and repeat the procedure.

We use the term "acceptable fit" to emphasize that we did not search the whole parameter space for pinpointing the set giving the best $\chi^{2}$ fit. This would not serve the purpose of this work, which can be summarized in the following: to calculate model spectra and lightcurves that can roughly account for both the time-averaged SED and the observed variability of PKS 2155304 and use them as a stepping stone for a qualitative comparison between the models. In any case, we verified that the general conclusions drawn of the present study are not strongly affected by the particular parameter values.

All model parameters could, in principle, be considered to vary with time in order to account for the observed variability.

3 This is partially true for leptohadronic models, since for particular parameter sets the system can exhibit limit cycle behaviour (Mastichiadis et al. 2005; Petropoulou \& Mastichiadis 2012b).
Changes in the injection rate, in the maximum electron energy, and in the Doppler factor are usually assumed while modelling flares (e.g. Coppi \& Aharonian 1999; Sikora et al. 2001; Krawczynski et al. 2002; Mastichiadis \& Moraitis 2008; Mastichiadis et al. 2013), as their impact on the SED is more direct. Other possibilities include variation of the magnetic field strength (e.g. Mastichiadis \& Kirk 1997; Moraitis \& Mastichiadis 2011), which might even lead to a change in the Compton dominance of the emission region in the context of SSC models. Here we test the hypothesis that the observed marginal variability in the low state is caused by variations of only two parameters, namely the maximum electron energy $\gamma_{\mathrm{e}, \max }$ and the injection compactness of electrons (and protons) $\ell_{\mathrm{e}, \mathrm{p}}^{\mathrm{inj}}$. Their temporal profile must be then determined, and for this, one can adopt one of the following approaches: (i) the phenomenological approach, where the functional form of the parameters is linked to the observed variability pattern in one or more energy bands; or (ii) the theoretical approach, where the variations are based upon a physical model for the acceleration and injection of particles in the emission region. In this work, we adopt the first approach which is after all the most commonly used.

In all three models, we assume that the variations imposed on $\gamma_{\mathrm{e} \text {,max }}$ of the first component are a scaled version of the observed X-ray variability. This hypothesis is physically motivated by the spectral hardening observed during episodes of increasing flux (see e.g. Fig. 1 in A09 and Fig. 2 in Barres de Almeida et al. 2014). Given the X-ray flux measurements at times $t_{\mathrm{obs}, \mathrm{i}}$, which are transformed in the comoving frame $\left(\tilde{t}_{\mathrm{i}}\right)$ using the Doppler factor value determined at step (1), we determine $\gamma_{\mathrm{e}, \max }$ at a previous time ${ }^{4} t_{\mathrm{i}}=\tilde{t}_{\mathrm{i}}-\Delta t$. Here, $\Delta t$ expresses the time in which the photon density in the emission region reacts to changes in the electron distribution and depends, in general, on the cooling and escape timescales of electrons. In what follows, we set $\Delta t=t_{\mathrm{cr}}$. We verified that slightly different values of $\Delta t$ do not alter the results. The intermediate values of $\gamma_{\mathrm{e}, \max }$ were then obtained by using a cubic spline interpolation scheme:

$\gamma_{\mathrm{e}, \max }(\tau)=\left\langle\gamma_{\mathrm{e}, \max }\right\rangle\left(\alpha_{1} \frac{F_{\mathrm{X}}(\tau)}{F_{\mathrm{X}}^{\max }}\right)^{\beta_{1}}$,

where $\left\langle\gamma_{\mathrm{e}, \max }\right\rangle$ is the value corresponding to the initial steady state; henceforth, the same will hold for all quantities enclosed by $\langle\cdots\rangle$. In the above expression $F_{\mathrm{X}}^{\max }=9.8 \times 10^{-11} \mathrm{erg} \mathrm{cm}^{-2} \mathrm{~s}^{-1}$ is the maximum flux in the $2-10 \mathrm{keV}$ energy band and $F_{\mathrm{X}}(\tau)$ is the result of a cubic spline interpolation of the observed $\mathrm{X}$-ray lightcurve expressed in terms of the dimensionless comoving time $\tau=t / t_{\mathrm{cr}}$. We set the day MJD 54703.5 as the zero time $(\tau=0)$ of our simulations.

In the 2-SSC model we assume that the variability in the optical and VHE gamma-rays is caused by changes in the maximum electron energy of the second component. Alternatively, one could argue that the optical/TeV variability can be explained by changes in the injection rate of electrons, but this would induce non-negligible $\mathrm{GeV}$ variability, which would contradict the observations, and so we do not consider this scenario further. Thus, $\gamma_{\mathrm{e}, \max }$ for the second component is modelled as

$\gamma_{\mathrm{e}, \max }(\tau)=\left\langle\gamma_{\mathrm{e}, \max }\right\rangle\left(\alpha_{2} \frac{F_{\mathrm{opt}}(\tau)}{F_{\mathrm{opt}}^{\max }}\right)^{\beta_{2}}$,

where $F_{\text {opt }}^{\max }=1.64 \times 10^{-10} \mathrm{erg} \mathrm{cm}^{-2} \mathrm{~s}^{-1}$ is the maximum flux measured in the $B V$ filters. We use subscripts 1,2 for the

4 The same procedure was followed for all parameters. 
Table 1. Parameter values for each of the three models discussed in text.

\begin{tabular}{|c|c|c|c|c|}
\hline \multirow{2}{*}{$\begin{array}{l}\text { Parameters for } \\
\text { initial steady state }\end{array}$} & \multirow[t]{2}{*}{ 1-SSC } & \multirow[t]{2}{*}{ LHs } & \multicolumn{2}{|c|}{ 2-SSC } \\
\hline & & & $1 \mathrm{st}$ & 2nd \\
\hline$B(\mathrm{G})$ & 0.5 & 40 & 20 & 0.1 \\
\hline$R(\mathrm{~cm})$ & $10^{16}$ & $10^{16}$ & $3 \times 10^{15}$ & $4.5 \times 10^{16}$ \\
\hline$\delta$ & 34 & 28 & 18 & 34 \\
\hline$\gamma_{\mathrm{e}, \min }{ }^{a}$ & $10^{3.6}$ & $10^{3.0}$ & $10^{3.8}$ & $10^{3.6}$ \\
\hline$\gamma_{\mathrm{e}, \max }$ & $10^{5.3}$ & $10^{4.7}$ & $10^{4.8}$ & $10^{4.1}$ \\
\hline$\ell_{\mathrm{e}}^{\mathrm{inj}}$ & $10^{-4.3}$ & $10^{-4.35}$ & $10^{-3.4}$ & $10^{-4.75}$ \\
\hline$s_{\mathrm{e}}$ & 2.4 & 2.6 & 2.7 & 1.7 \\
\hline$q_{\mathrm{e}}$ & 1.0 & 2.0 & 1.0 & 1.0 \\
\hline$\gamma_{\mathrm{p}, \min }$ & - & $10^{7}$ & - & - \\
\hline$\gamma_{\mathrm{p}, \max }{ }^{b}$ & - & $10^{9.9}$ & - & - \\
\hline$\ell_{\mathrm{p}}^{\mathrm{inj}}$ & - & $10^{-6.4}$ & - & - \\
\hline$s_{\mathrm{p}}$ & - & 2.4 & - & - \\
\hline$q_{\mathrm{p}}$ & - & 2.0 & - & - \\
\hline \multicolumn{5}{|l|}{$\begin{array}{l}\text { Parameters for } \\
\text { variability }\end{array}$} \\
\hline$\alpha_{1}, \beta_{1}$ & $1.8,1.0$ & $2.0,1.8$ & \multicolumn{2}{|c|}{$2.1,1.8$} \\
\hline$\alpha_{2}, \beta_{2}$ & - & - & \multicolumn{2}{|c|}{$2.5,1.1$} \\
\hline$f_{\mathrm{e}}, f_{\mathrm{p}}$ & - & $0.6,0.6$ & \multicolumn{2}{|c|}{-} \\
\hline$g_{\mathrm{e}}, g_{\mathrm{p}}$ & - & $0.0,-0.6$ & \multicolumn{2}{|c|}{-} \\
\hline
\end{tabular}

Notes. ${ }^{(a)}$ These values correspond to the quantities enclosed by $\langle\cdots\rangle$ in text. ${ }^{(b)}$ This value satisfies the Hillas criterion, i.e. $\gamma_{\mathrm{p}, \max }<e B R / m_{\mathrm{p}} c^{2} \simeq$ $1.5 \times 10^{11}$.

parameters $\alpha, \beta$ to refer to the first and second components, respectively. We note that if the injection compactness is kept fixed while $\gamma_{\mathrm{e}, \max }$ varies according to Eqs. (4) or (5), then the injection rate of particles is also variable (see Eqs. (1)-(3)).

Contrary to $\gamma_{\mathrm{e}, \max }$ that varied in all three models, the injection compactness of primary particles was assumed to be time-dependent only in the LHs scenario. In this case, the emission from the optical up to X-rays is the result of electron synchrotron radiation, whereas the proton synchrotron component dominates in the gamma-ray energy band. Because of the tight correlation between the optical and $\mathrm{TeV}$ fluxes we chose to model $\ell_{\mathrm{e}}^{\mathrm{inj}}$ and $\ell_{\mathrm{p}}^{\mathrm{inj}}$ according to the variability pattern observed in the optical filters:

$\ell_{\mathrm{e}, \mathrm{p}}^{\mathrm{inj}}=\left\langle\ell_{\mathrm{e}, \mathrm{p}}^{\mathrm{inj}}\right\rangle\left(\frac{1}{f_{\mathrm{e}, \mathrm{p}}} \frac{F_{\mathrm{opt}}}{F_{\mathrm{opt}}^{\mathrm{max}}}+g_{\mathrm{e}, \mathrm{p}}\right)$.

In total, we introduced eight free parameters, namely $\alpha_{1,2}, \beta_{1,2}$, $f_{\mathrm{e}, \mathrm{p}}$ and $g_{\mathrm{e}, \mathrm{p}}$, to account for the observed variability. All the parameter values, including those leading to the steady state solution that served as an initial condition for our calculations are summarized in Table 1.

\section{Results}

In the following paragraphs we present snapshots of the SED and lightcurves for each of the models discussed in the previous section and comment also on the pros and cons of each model. In all cases, the VHE part of the derived gamma-ray spectra has been absorbed using the EBL model (Model C) by Finke et al. (2010).

\subsection{One-component SSC model (1-SSC)}

Figure 1 shows the $\mathrm{X}$-ray lightcurve (top panel) derived from the model for a variable $\gamma_{\mathrm{e}, \max }$ (Eq. (4)) with $\alpha_{1}=1.8$ and $\beta_{1}=1.0$
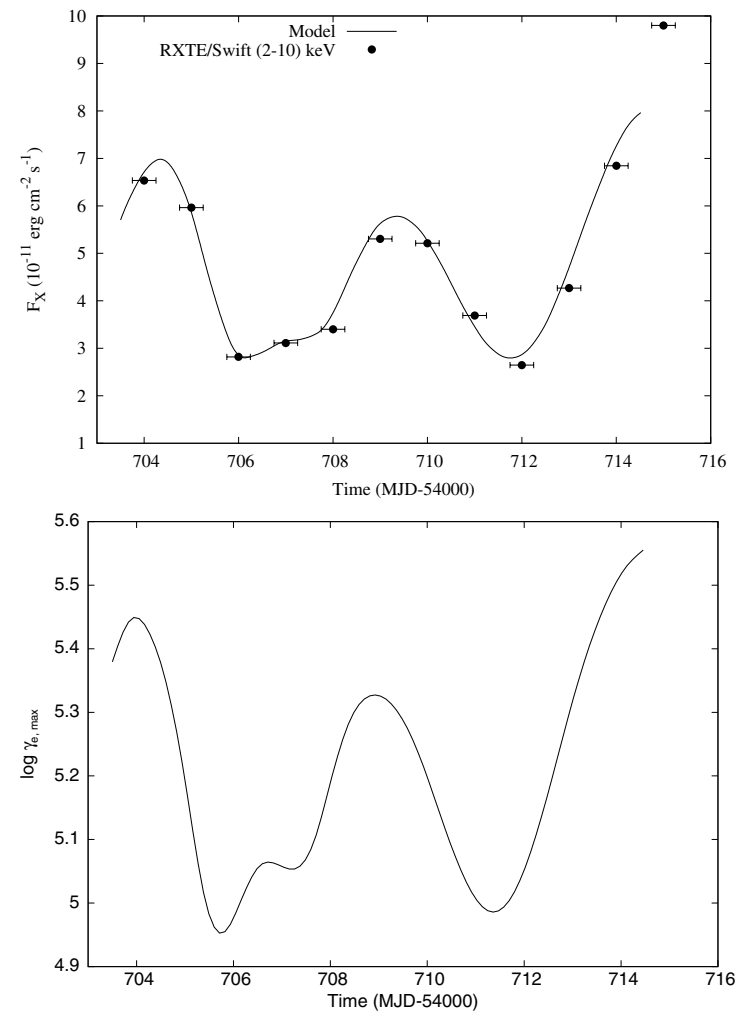

Fig. 1. Top panel: X-ray (2-10 keV) lightcurve from the 2008 campaign (A09) along with the SSC model fit. Bottom panel: variations of $\gamma_{\mathrm{e}, \max }$ used as an input to the numerical code.

(bottom panel). This choice of parameters leads to small amplitude variations of $\gamma_{\mathrm{e}, \max }$, i.e. it varies at most by a factor of 4 between MJD 54706 and MJD 54715.

The X-ray observations (points) are satisfactorily reproduced by the model except for the flux at MJD 54715, which is higher by a factor of 1.22 compared to our model. At the same date the X-ray spectrum of PKS 2155-304 was found to be harder with respect to the previous days, having a photon index $\Gamma_{X} \simeq$ 2.3 (A09). Our model spectra, on the other hand, have a photon index of $\sim 2.4$. Thus, the ratio of the observed and the model derived $\mathrm{X}$-ray fluxes in the range $2-10 \mathrm{keV}$ is $\sim 0.4 / 0.3$, i.e. close to the difference seen in Fig. 1. If we had allowed the power-law index $s_{\mathrm{e}}$ to vary with time in addition to $\gamma_{\mathrm{e}, \max }$, the fit of the X-ray lightcurve would have been improved. We do not consider this case here because the number of free parameters is already large and none of the general conclusions of the analysis would be altered. This is a general remark that applies to the other models, too.

Snapshots of the photon spectra corresponding to MJD 54 706, 54 709, and 54715 are shown in Fig. 2, where all data points are taken from A09. Although the model describes fairly well the X-ray behaviour, it fails to reproduce the $\mathrm{TeV}$ and optical variability, which can be seen already from the SED snapshots in Fig. 2. Electrons emitting synchrotron radiation in the X-ray energy band lie close to the upper cutoff of the distribution, i.e. $\gamma_{X} \simeq 10^{5}$ for $\epsilon_{X}=2 \mathrm{keV}, \delta=34$ and $B=0.5 \mathrm{G}$. The same electrons will upscatter the $X$-ray synchrotron photons in the Klein-Nishina regime, since $\gamma_{X} \epsilon_{X} /\left(\delta m_{\mathrm{e}} c^{2}\right) \gg(3 / 4)$. Thus, the upscattered photons will be produced at $\mathrm{TeV}$ energies, i.e. $\epsilon_{\gamma} \simeq \delta \gamma_{X} m_{\mathrm{e}} c^{2} \simeq 1.7 \mathrm{TeV}$, and strong correlation between the $\mathrm{TeV}$ and $\mathrm{X}$-ray fluxes is expected. 


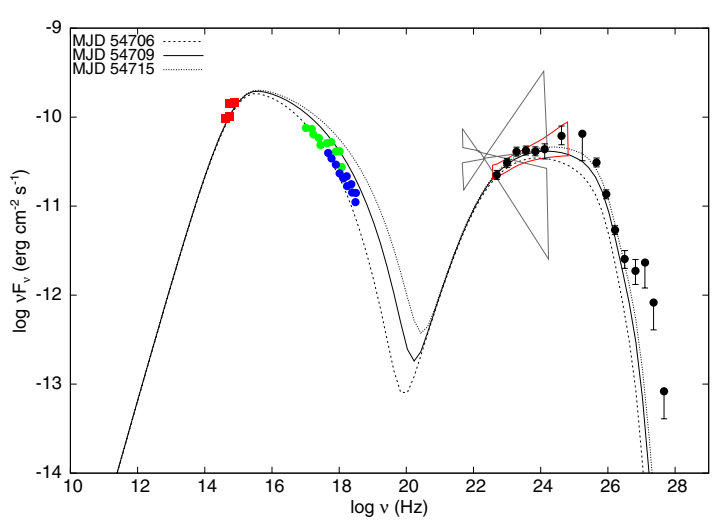

Fig. 2. Snapshots of multiwavelength (MW) spectra during the period MJD 54704-54715 in the context of the one-zone SSC model. All data points are from A09. From low to high energies: optical measurements (red points) from ATOM, combined RXTE and Swift X-ray measurements (green and blue points), and gamma-ray observations (black points) by Fermi-LAT and H.E.S.S. in the GeV and TeV energy bands, respectively. The red butterfly is the actual Fermi spectrum for the period MJD 54704-54715 and the grey ones show EGRET measurements.

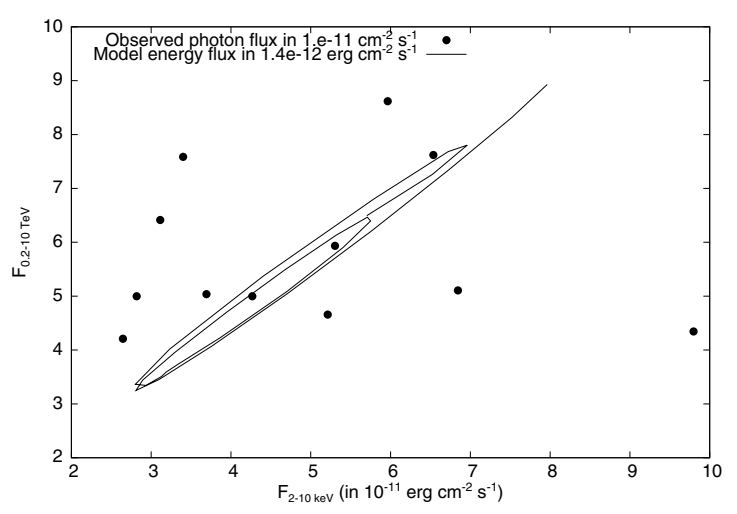

Fig. 3. Gamma-ray (energy or photon) flux in $0.2-10 \mathrm{TeV}$ against the $2-10 \mathrm{keV} \mathrm{X}$-ray flux. The observations are shown with symbols, whereas the result of the 1-SSC model is plotted with a solid line. As $\mathrm{TeV}$ flux, we use the observed photon flux normalized to $10^{-11} \mathrm{~cm}^{-2} \mathrm{~s}^{-1}$ and the model derived energy flux in $0.2-10 \mathrm{TeV}$.

This is exemplified in Fig. 3 where we plot the TeV flux against the X-ray flux obtained by our model (line) and by the observations (symbols). For the plot, we used the photon flux observed in $0.2-10 \mathrm{TeV}$ with H.E.S.S. and the energy flux calculated by the model. These are normalized to the values marked on the plot. The plot shows that the observed X-ray and TeV fluxes are not correlated, in contrast to the model prediction. By applying a Pearson's correlation test to the model derived fluxes we find, indeed, a strong correlation with the correlation coefficient being $r=0.99$. This is a robust prediction of the 1-SSC model, which contradicts the observed loose correlation. We find also that the TeV flux scales linearly to the X-ray one in agreement to the analysis by Katarzyński et al. (2005). The authors showed that the relation between the $\mathrm{X}$-ray and $\mathrm{TeV}$ fluxes above the respective peaks of the SED is usually less than quadratic.

Moreover, the variation of $\gamma_{\mathrm{e}, \max }$ alone cannot account for the optical variability. For the adopted parameters, the cooling Lorentz factor of electrons is $\gamma_{\mathrm{e}, \mathrm{c}} \approx 9 \times 10^{3}>\gamma_{\mathrm{e}, \min }$ and their typical synchrotron frequency $v_{\mathrm{c}} \simeq 4 \times 10^{15} \mathrm{~Hz}$. Thus, it is the low-energy part of the synchrotron spectrum
$\left(F_{v} \propto v^{1 / 3}\right)$ that falls in the optical window and is unaffected by the small-amplitude variations induced at the high-energy part of the synchrotron spectrum. One could think of a scenario where the electron injection compactness would vary according to the optical variability. In this case, however, a larger variation of $\gamma_{\mathrm{e}, \max }$ would be required. This can be understood as follows: whenever the X-ray flux increases significantly, the optical emission is at a low level. Thus, in order to compensate for the low $\ell_{\mathrm{e}}^{\mathrm{inj}}$ value, one would have to assume larger variations to $\gamma_{\mathrm{e}, \max }$. As a result, the $\mathrm{TeV}$ flux would show the same temporal pattern as the X-rays. For large enough variations of $\gamma_{\mathrm{e}, \max }$, the model would predict spectral variability in the $\mathrm{TeV}$ energy band even in the presence of Klein-Nishina cutoff effects, which are once again excluded from the observations. We do not investigate in any more detail this hypothesis, since the $\mathrm{X}$-ray/TeV correlation would still be present.

Summarizing, the one-component SSC model can explain both the time-averaged SED and the X-ray variability despite its simplicity and the small number of free parameters. However, its main drawback is the robust prediction of a tight correlation between the $\mathrm{X}$-rays and $\mathrm{TeV}$ gamma-rays. Interestingly, the usual SSC model was also challenged, for different reasons, by an exceptional gamma-ray flaring event of PKS 2155-304 in 2006 (Aharonian et al. 2009a).

\subsection{Proton synchrotron model (LHs)}

In this scenario the low energy bump (optical up to X-rays) is attributed to synchrotron radiation of relativistic electrons, whereas the high-energy bump ( $\mathrm{GeV}$ up to $\mathrm{TeV}$ gamma-rays) is considered to be the synchrotron emission of a relativistic proton population. Although the emission over the whole energy range originates from the same region, the LHs model can be thought of as a two-component emission model because of the two independent particle populations contributing to the lowand high-energy parts of the spectrum. The number of free parameters required for modelling steady-state photon emission increases from eight in the 1-SSC model to thirteen (see also Table 1). To these one has to include six additional parameter $\left(\alpha_{1,2}, f_{\mathrm{p}, \mathrm{e}}\right.$ and $\left.g_{\mathrm{p}, \mathrm{e}}\right)$ for modelling the variability in the optical, $\mathrm{X}$-rays and $\mathrm{TeV}$ gamma-rays. In panels of Fig. 4 from left to right we present the observed lightcurves (points) along with the model results (solid lines) for the optical, X-ray and TeV energy bands, respectively. The H.E.S.S. lightcurve in A09 was given in units of photon count rate per effective area of the detector, and for this reason a direct comparison to the flux calculated with our model could not be made. Given that we do take into account the time-averaged SED in our analysis, it is sufficient to compare the relative variations, i.e. $F_{\mathrm{TeV}} / \bar{F}_{\mathrm{TeV}}$ to $\dot{N}_{\mathrm{TeV}} / \overline{\dot{N}}_{\mathrm{TeV}}$, where quantities with a bar denote the average values over the twelve day period and $\dot{N}_{\mathrm{TeV}}$ is the observed photon count rate per effective area (see also Fig. 1 in A09). The model lightcurve can reproduce the observed variations apart from the first two data points of H.E.S.S. This is not unexpected, since we modelled $\ell_{\mathrm{e}}^{\mathrm{inj}}$ according to the optical variability, which does not correlate with the $\mathrm{TeV}$ lightcurve at least for the first two days (MJD 54 704-54 705).

We note also that the above comparison is possible only in the absence of spectral variation. For this, we did not attempted a similar comparison to the Fermi data that exhibit spectral changes, especially in the first three days, although the flux remains approximately constant. However, we calculated the $0.2-300 \mathrm{GeV}$ flux of the model and found that it varies at 

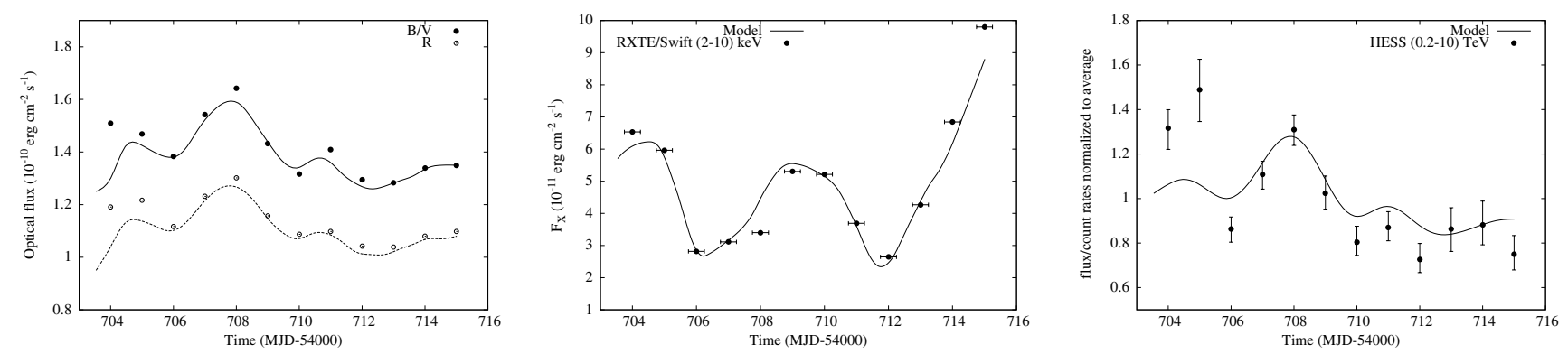

Fig. 4. From left to right: optical, X-ray and TeV lightcurves calculated using the LHs model. For the parameters see Table 1.
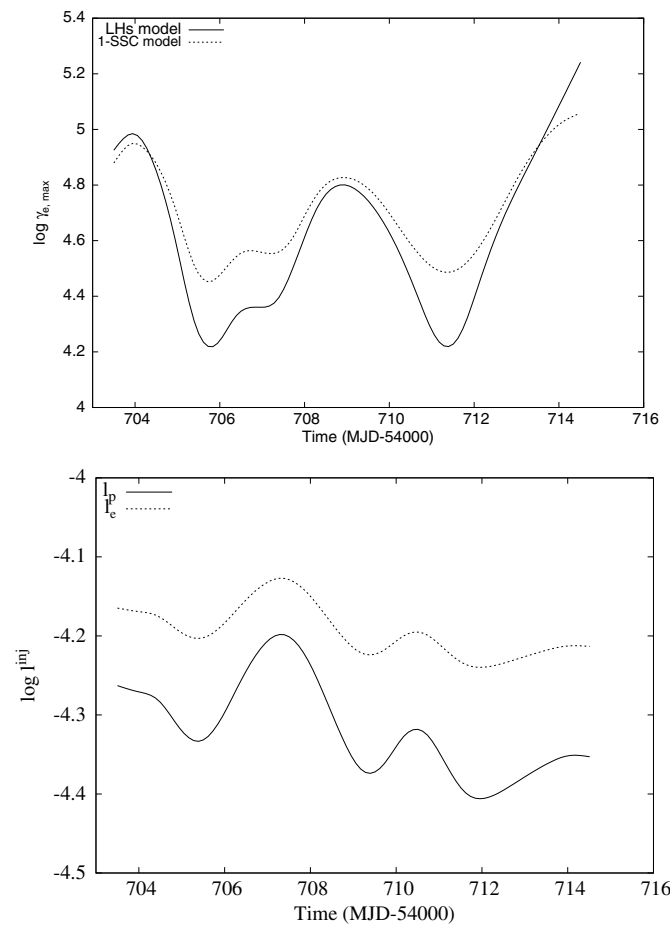

Fig. 5. Top panel: comparison of $\gamma_{\mathrm{e}, \max }$ used for the LHs (solid line) and 1-SSC (dashed line) models; for comparison reasons, the latter is shifted by a factor of -0.5 in logarithm. Bottom panel: $\ell_{\mathrm{p}}^{\text {inj }}$ (solid line) and $\ell_{\mathrm{e}}^{\mathrm{inj}}$ (dashed line) as a function of time where the former is shifted by +2.3 logarithmic units.

most by a factor of 1.6 , i.e. it is compatible with a constant value. Figure 6 shows snapshots of the SEDs obtained in the LHs scenario, where it becomes evident that the GeV part of the SEDs shows no spectral variation. To account for the observed spectral variations in the $\mathrm{GeV}$ band, one should treat an additional model parameter, e.g. the power-law index $s_{\mathrm{p}}$ of the proton distribution, as time dependent.

The above results were obtained by varying $\gamma_{\mathrm{e}, \max }$ and $\ell_{\mathrm{e}, \mathrm{p}}^{\mathrm{inj}}$ as shown in the top and bottom panels of Fig. 5. The relative change of $\gamma_{\mathrm{e}, \max }$ in the LHs model (solid line) is larger compared to that required by the 1-SSC model (dashed line), mainly because of the steeper electron distribution assumed $\left(s_{\mathrm{e}}=2.6\right)$. Besides $\gamma_{\mathrm{e}, \max }$, which changes by an order of magnitude, the variations of the injection compactnesses are small.

Summarizing, the LHs model can account for (i) the observed flux variability from the optical up to the TeV energy band; (ii) the approximately constant $\mathrm{GeV}$ flux; and (iii) the absence of correlation between the $\mathrm{X}$-rays and $\mathrm{TeV}$ gamma-rays but it cannot explain the spectral variability in $\mathrm{GeV}$ gamma-rays unless the proton distribution slope changes. In total, it requires three (or four) model parameters to be functions of time.

\subsection{Two-component SSC model (2-SSC)}

The uncorrelated variability between the optical and X-ray fluxes is one of the most intriguing results of the 2008 campaign. Motivated by the difficulty to explain the presence of no correlation in the context of a homogeneous one-component emission model (some of the problems were already discussed in Sects. 4.1 and 4.2), we considered a two-component SSC scenario, where the total emission originates from two physically distinct regions. Synchrotron radiation from the first one accounts only for the X-ray flux, whereas the emission from the second component dominates in the optical (synchrotron) and gamma-ray (SSC) energy bands. The above physical setup implies that the parameters describing the first emission region should be such as to suppress its SSC emission. Moreover, we treat both regions independently by assuming that only the synchrotron photons produced within the same region serve as targets for inverse Compton scattering. For the parameters used here, this assumption is valid as long as the separation $r_{12}$ between the two regions is a few times the size of the larger component, i.e. $r_{12} \gtrsim 6 \times 10^{16} \mathrm{~cm}$ (for more details, see Appendix).

Figure 7 shows snapshots of SEDs for both components covering the whole observing period. The SED of the first component (black lines) is clearly synchrotron dominated with a peak at $\sim 0.4 \mathrm{keV}$ and a SSC peak luminosity being approximately $1 \%$ of the synchrotron peak luminosity. The SED of the second component (grey lines) has the usual double-humped shape with the synchrotron and SSC spectra peaking at far$\mathrm{UV}$ and $\sim \mathrm{GeV}$ energies, respectively. Because of the underlying electron distribution, which is hard and spans over only one decade in energy (see Table 1), the two bumps of the SED appear narrow with large curvature (for relevant detailed discussion, see Massaro et al. 2006). This results in the following: the second component contributes only a small fraction to the $2-10 \mathrm{keV}$ flux, since its integrated $\mathrm{X}$-ray flux does not exceed the value $3 \times 10^{-12} \mathrm{erg} \mathrm{cm}^{-2} \mathrm{~s}^{-1}$ and the model slightly underpredicts the H.E.S.S. flux. This underprediction could be resolved by assuming a broader electron distribution. This would be, however, problematic, as the synchrotron spectra would dominate the emission in the X-rays and thus destroy the loose correlation between them and the VHE gamma-rays.

The differences in the spectral shape reflect the differences in the underlying physical quantities describing each component (see Table 1), in contrast to Barres de Almeida et al. (2014) where similar values of $\delta, B$ and $R$ were attributed to both components. In our analysis, the first component is more compact 


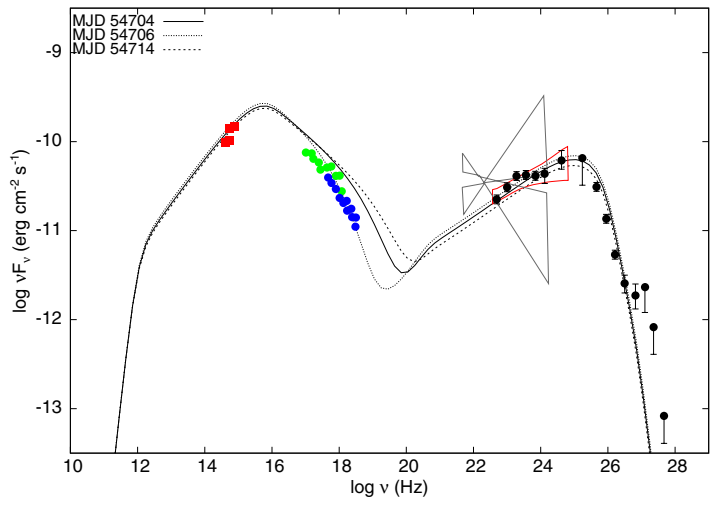

Fig. 6. Snapshots of the SED obtained in the context of the LHs model for MJD 54 704, 54706 and 54714.

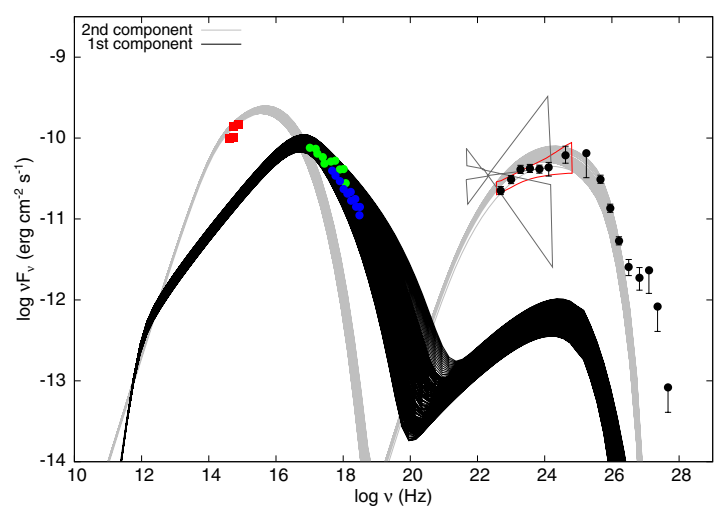

Fig. 7. MW photon spectra calculated using the 2-SSC model for the whole period MJD 54 704-54 715 that demonstrate the difference in the range of variance of the two components. The emission from the first and second components are plotted with black and grey lines, respectively. For the parameters used, see Table 1.

because of its smaller size and its lower Doppler factor and contains a stronger magnetic field. One can relate the location $r_{i}$ of each component to its radius $R_{i}$ as $R_{i} \approx r_{i} \theta_{i}$, where $\theta_{i}$ is the opening angle of each region, which typically is smaller than the opening angle of the jet. In the limit where $\delta_{i} \simeq \Gamma_{i}$ and under the assumption of collimation ${ }^{5}$, i.e. $\Gamma_{i} \theta_{i}<1$, one finds that $r_{i} \gtrsim R_{i} \delta_{i}$. Using the values of Table 1 we find that $r_{1} \gtrsim 5.4 \times 10^{16} \mathrm{~cm}$ and $r_{2} \gtrsim 1.5 \times 10^{18} \mathrm{~cm}$, i.e. the first and second components should be placed at the sub-pc and pc-scale jet, respectively. We also note that the synchrotron self-absorption frequency of the first component appears at $\sim 10^{12} \mathrm{~Hz}$, i.e. well above the $\mathrm{GHz}$ radio band. Thus, one could go one step further and argue that any radio variability observed in the low state should be correlated with the optical and VHE gammarays but not with the X-rays; such observational evidence, however, is still lacking. For a better comparison of the energetics, we summarize in Table 2 the average ${ }^{6}$ photon and electron energy densities together with the magnetic energy density for the two-components as measured in their respective rest frames. For the first and second components we find $\bar{u}_{\mathrm{e}} \ll \bar{u}_{\gamma} \ll u_{B}$ and $\bar{u}_{\gamma} \lesssim u_{B}<\bar{u}_{\mathrm{e}}$, respectively.

Besides the differences in their physical properties and emitted SEDs, the two components show also differences in their

\footnotetext{
5 For a detailed discussion with specific examples from FSRQs, see Nalewajko et al. (2014).

6 We calculate the average value over the period MJD 54 704-54 715.
}

Table 2. Photon $\left(\bar{u}_{\gamma}\right)$, electron $\left(\bar{u}_{\mathrm{e}}\right)$ and magnetic $\left(u_{B}\right)$ energy densities for the 2-SSC scenario.

\begin{tabular}{lccc}
\hline \hline & $\bar{u}_{\gamma}\left(\mathrm{erg} / \mathrm{cm}^{3}\right)$ & $\bar{u}_{\mathrm{e}}\left(\mathrm{erg} / \mathrm{cm}^{3}\right)$ & $u_{B}\left(\mathrm{erg} / \mathrm{cm}^{3}\right)$ \\
\hline $1 \mathrm{st}$ & $4 \times 10^{-1}$ & $4.7 \times 10^{-3}$ & 16 \\
2nd & $5 \times 10^{-4}$ & $3 \times 10^{-3}$ & $4 \times 10^{-4}$ \\
\hline
\end{tabular}

Notes. For the first two quantities we present their average values over the period MJD 54 704-54 715.

variability properties. This can be seen by the clustering of all the snapshots of the second component in contrast to the wider range of variance of the first component. To derive the above SEDs we modelled $\gamma_{\mathrm{e}, \max }$ according to Eqs. (4) and (5) for the first and second component, respectively. In this way we can ensure once again the correlation between the optical and TeV fluxes, while the X-ray emission will be uncorrelated with both of them.

The lightcurves obtained from the 2-SSC model are presented in Fig. 8. The abrupt increase in the model derived optical and TeV fluxes just before MJD 54704 has to do with the initial steady state, and in this sense it could be avoided for another choice of initial conditions. For this, we do not consider the early part in the discussion of our results. The TeV lightcurve is the less satisfactory result of the 2-SSC model, since it cannot reproduce the increasing trend of the first two days, for the same reasons as in the LHs scenario, and it overestimates the ratio $F_{\mathrm{TeV}} / \bar{F}_{\mathrm{TeV}}$ between the days $54706-54708$. We note that given the freedom that the multiparameter models provide, a more detailed search of the parameter space might result in a better fit.

Summarizing, we showed that the 2-SSC model can satisfactorily explain (i) the time-averaged SED; (ii) the optical and $\mathrm{X}$-ray lightcurves; (iii) the optical/TeV correlation; and (iv) the absence of correlation between the X-rays and TeV gamma-rays, yet the model requires a large number of free parameters and suggests the presence of two distinct regions, being energetically different, within the same jet, something that is difficult to reconcile with the present understanding of jet physics.

\subsubsection{A flaring event}

Here we present two indicative examples of flaring events because of the activation of one of the emitting components in the context of the 2-SSC scenario. In particular, we assume that the period of low activity (MJD 54 704-54715) is followed by an active period of the second component lasting nine days. To model the high state period we introduced Lorentzian variations to either $\gamma_{\mathrm{e}, \max }$ or $\ell_{\mathrm{e}}^{\mathrm{inj}}$. All the parameters, except for those that have to do with the variability, are the same as in Table 1.

We investigated the following cases:

- Flare A: $\gamma_{\mathrm{e}, \max }$ is varying according to

$$
\frac{\gamma_{\mathrm{e}, \max }(\tau)}{\gamma_{\mathrm{e}, \max }\left(\tau_{\mathrm{e}}\right)}=\frac{\tau_{\mathrm{p}}^{2}+(G / 2)^{2}}{\left(\tau-\tau_{\mathrm{p}}\right)^{2}+(G / 2)^{2}}, \tau \geq \tau_{\mathrm{e}},
$$

where $\tau_{\mathrm{e}}$ is end time of the low state period (in $t_{\mathrm{cr}}$ units), which we set equal to zero, $G=25, \tau_{\mathrm{p}}=25$ and $\gamma_{\mathrm{e}, \max }\left(\tau_{\mathrm{e}}\right)=$ $10^{4.5}$. The increase in $\gamma_{\mathrm{e}, \max }$ leads to correlated optical, X-ray and gamma-ray flares.

- Flare $\mathrm{B}: \ell_{\mathrm{e}}^{\mathrm{inj}}$ is varying according to

$$
\frac{\ell_{\mathrm{e}}^{\mathrm{inj}}(\tau)}{\ell_{\mathrm{e}}^{\mathrm{inj}}\left(\tau_{\mathrm{e}}\right)}=\frac{\tau_{\mathrm{p}}^{2}+(G / 2)^{2}}{\left(\tau-\tau_{\mathrm{p}}\right)^{2}+(G / 2)^{2}}, \tau \geq \tau_{\mathrm{e}}
$$



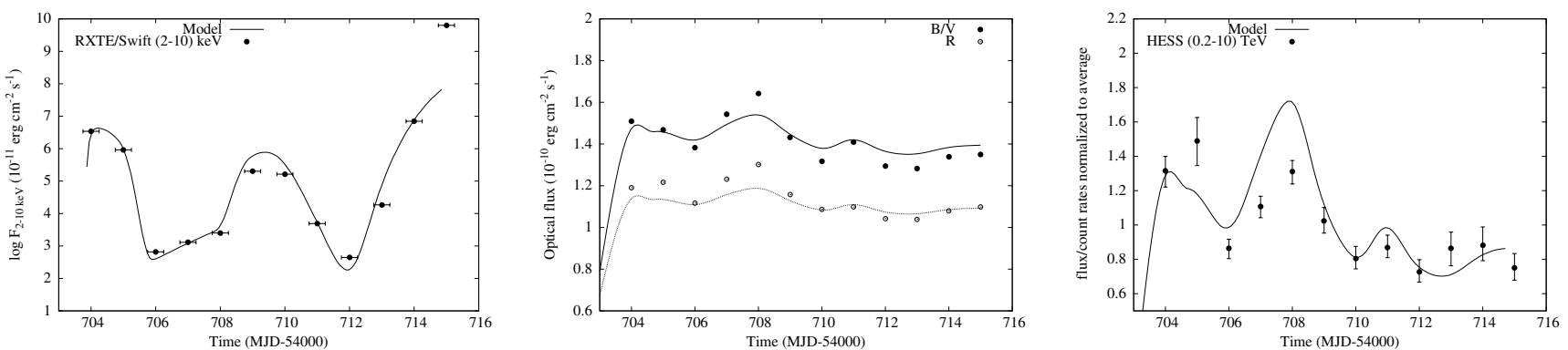

Fig. 8. Same as in Fig. 4 but for the two-component SSC model.
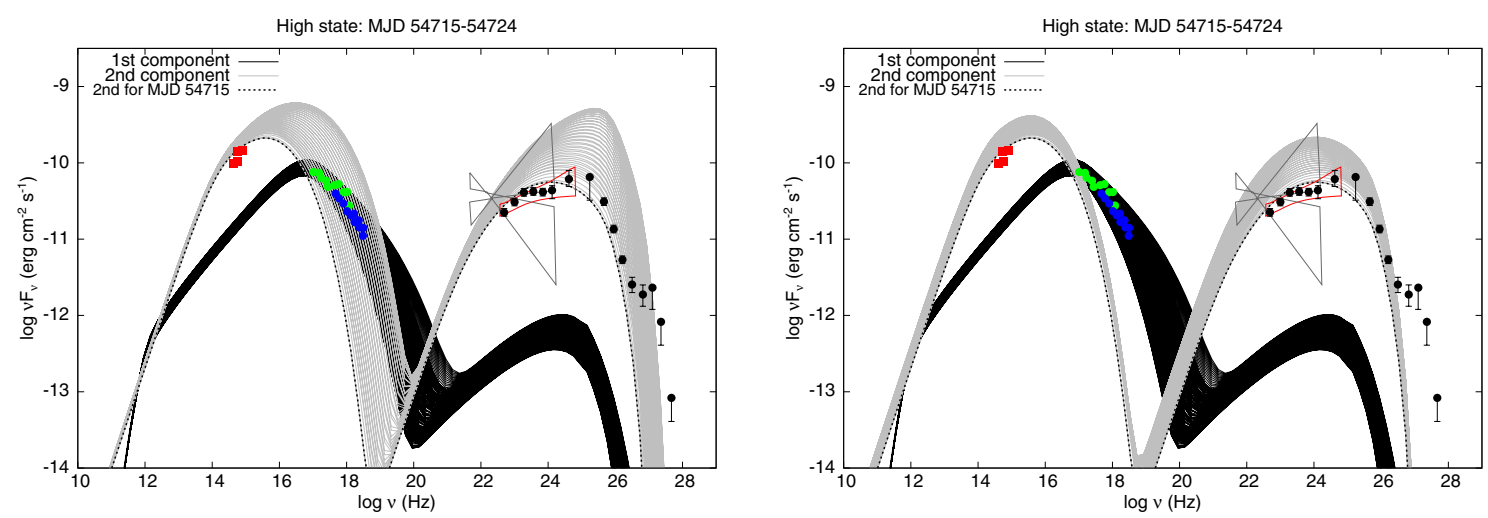

Fig. 9. Time-dependent SEDs (grey lines) of a fiducial high-state period starting after MJD 54715 for the two scenarios discussed in text. The results for Flares A and B are shown in the left and right panels, respectively. In both panels the last SED of the low-state for the second component is also shown with black dashed line. The first component retains the same small-amplitude variability of the low-state period (black lines). For comparison reasons, the observations of the low state period are also shown.
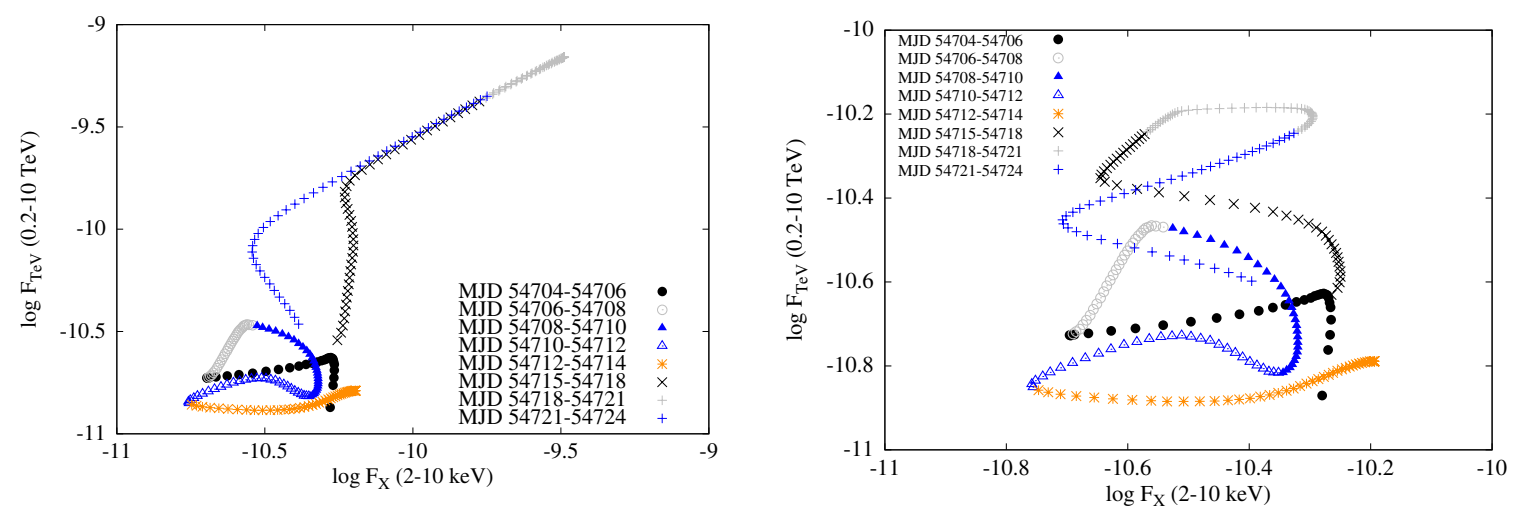

Fig. 10. $F_{\mathrm{TeV}}-F_{\mathrm{X}}$ plane in logarithmic scale for the periods of low and high activity. The results for flares A and $\mathrm{B}$ are shown in the left and right panels, respectively. Different symbols are used to denote different phases of the lightcurve.

where $G=50, \tau_{\mathrm{p}}=25$ and $\ell_{\mathrm{e}}^{\mathrm{inj}}\left(\tau_{\mathrm{e}}\right)=10^{-4.75}$. The increase in the electron compactness results in a correlated optical/gamma-ray flare.

Our results are summarized in Fig. 9 where the left and right panels show time-dependent SEDs obtained for Flares A and B, respectively. Even though $\gamma_{\mathrm{e}, \max }$ changes only by a factor of 4 in Flare A, the luminosity of both the synchrotron and SSC components increases significantly, because of the hard energy spectrum of the electron distribution. At the beginning of the active period, the X-ray emission is still dominated by the first component but as $\gamma_{\mathrm{e} \text {,max }}$ gradually increases, both components, each of them having its own temporal behaviour, contribute to the X-ray flux. This may lead to interesting patterns in the $F_{\mathrm{TeV}}-F_{\mathrm{X}}$ plane, as it can be seen in the left panel of Fig. 10. One can distinguish between phases of strong correlation, e.g. MJD 54 718-54721, but also periods where the two are anti-correlated. The reason is that the X-ray flux is the sum of both components, each of them contributing the most to the total flux at different periods, while having different lightcurve shapes.

In flare B the contribution of each component to the overall SED during the high-state period is clear. Such scenarios could explain correlated optical and gamma-ray activity, with the amplitude of optical flares being typically smaller than the one in gamma-rays (see e.g. right panel in Fig. 9). The flux-flux 
diagram for the low-state and high-state periods is shown in the right panel of Fig. 10. We find no significant correlation when we take into account the whole period of 20 days; the Pearson's correlation coefficient is $r=-0.07$ with probability $P<24 \%$ of a chance correlation. The correlation may be enhanced, however, if we focus on subsets of 1-3 days. Thus, even in scenarios where the $\mathrm{TeV}$ and $\mathrm{X}$-ray fluxes originate from different components, each of them having its own temporal behaviour, a strong correlation between the two may be obtained.

One could think of scenarios where the first component is the active one, thus leading to correlated X-ray/gamma-ray variability and an approximately constant optical flux. For the adopted parameter values, the SSC emission of the first component is strongly suppressed and changes of just $\ell_{\mathrm{e}}^{\mathrm{inj}}$ and/or $\gamma_{\mathrm{e}, \max }$ would not suffice to enhance the SSC emission to the observed flux levels. Thus, this flaring scenario would require changes in the B-field and/or size of the first emitting region and should be studied in more detail in a future work.

\section{Discussion}

The high quality of observational data has allowed us to attempt both spectral and temporal fits to the observations of blazar PKS 2155-304 while being in a low state (25 August6 September 2008). We applied both leptonic and leptohadronic scenarios and focused on variants that were successful in fitting the time-averaged SED.

We showed that the one-component SSC model can explain the time-averaged SED as well as the X-ray variability. However, it predicts a tight correlation between the X-rays and $\mathrm{TeV}$ gamma-rays, which contradicts the loose correlation observed in the low state of PKS 2155-304. For this reason, we suggest the 1-SSC model to be the least plausible among the three scenarios, although its simplicity makes it the most attractive. We have shown that two-component emission models, namely the LHs and 2-SSC models, are more adequite in reproducing the observed SED and lightcurves, at the cost of a large number of free parameters. We note also that the aforementioned models have also their share of weak points, the most important being the disagreement between the model and observed TeV lightcurves at early times (MJD 54 704-54 705). Other variants of the leptohadronic model, such as the photopion model where the gamma-ray emission is attributed to the synchrotron radiation of pairs produced through pion decay, were not discussed here because of their intrinsic TeV/X-ray flux correlation (Mastichiadis et al. 2013).

In all three scenarios, the observed marginal variability was modelled by assuming that the maximum energy of electrons and/or the injection compactness of particles are variable, with the required variations being a scaled version of the lightcurve in one or more energy bands (see Sect. 3). In principle, the changes in both $\ell_{\mathrm{e}, \mathrm{p}}^{\mathrm{inj}}$ and $\gamma_{\mathrm{e}, \max }$ could be related to variations of the conditions in the acceleration zone, which in our framework, is considered to be a black box acting as a reservoir of accelerated particles for the emission (radiation) zone. In the case of shock acceleration for example, the encounter of the shock with a region of higher (lower) density could result in an increase (decrease) in the injection rate of particles in the acceleration region, and subsequently, in the emission region. Variations of the maximum energy of accelerated particles usually imply changes in the acceleration $\left(t_{\mathrm{acc}}\right)$ or/and energy loss $\left(t_{\text {loss }}\right)$ timescales, since $\gamma_{\max }$ is the energy where the energy loss and energy gain rates become equal (see e.g. Dermer \& Humi 2001; Petropoulou et al. 2014). For example, in the simplest scenario where particles are shock accelerated at the Bohm rate and lose energy through synchrotron radiation, particle acceleration saturates at $\gamma_{\mathrm{e}, \max }=\left(6 \pi e / \sigma_{\mathrm{T}} B_{0}\right)^{1 / 2}$, with $B_{0}$ being the magnetic field strength in the acceleration zone and $e$ the electron charge. Doubling of $\gamma_{\mathrm{e}, \max }$ would thus require a decrease of $B_{0}$ by a factor of 4 . The dependence of $\gamma_{\mathrm{e}, \max }$ on the various physical quantities, however, is critically determined by the acceleration mechanism at work (for possible acceleration mechanisms and their respective $t_{\text {acc }}$, see Tammi \& Duffy (2009) and references therein). Thus, an interpretation of the derived parameter variations in the context of a particular physical mechanism lies out of the scope of the present work.

The emission of blazars in high (flaring) states is what usually draws the attention, since modelling of high states may give insight to the properties of the radiating particles (for a relevant discussion, see e.g. Aharonian et al. 2009a). Our timedependent analysis showed, however, that we can deduce information about the properties of the emission region(s) even when the observations correspond to periods of low activity. The particular set of observations has been also recently discussed by Barres de Almeida et al. (2014) in a different context. This is a great opportunity for a qualitative comparison of the results obtained by two different approaches for the same dataset and for the same source. Starting from a different basis, namely optical polarization measurements during the 54711-54715 period, Barres de Almeida et al. (2014) conclude that the X-ray emission must originate from the same component that is responsible for the variations seen in the polarization of optical data. At the same time, this component should be hidden by the second one both in the optical and gamma-ray energy bands - for comparison see Fig. 7 in this work and Fig. 3 in Barres de Almeida et al. (2014). Given that we did not aim to derive the best-fit parameter values, a quantitative comparison lies out of the scope of the present study.

Although the results of our time-resolved analysis do not favour the LHs over the 2-SSC model, they suggest that the SED of PKS 2155-304 in a low state is composed by the emission of at least two components, which correspond to either different particle populations or spatially different emission regions. In this scenario a loose correlation between the various energy bands is expected whenever the source is not active, whereas if the source enters a high state where only one of the components lights up, tight correlations should be expected. Such correlations are quite common in flaring blazars, e.g. Mrk 421 (Fossati et al. 2008), and this was indeed the case during the exceptional flaring event of PKS 2155-304 in 2006 (Aharonian et al. 2009a), where a strong correlation between the X-rays and TeV gammaray was observed (for more details, see Aharonian et al. 2009a).

Concluding, the time-resolved analysis of the MW observations of PKS 2155-304 between August and September 2008, points that the quiescent emission of a blazar may result from a superposition of the radiation from different components, whereas the high state emission may still be the result of a single component. This demonstrates the importance of contemporeneous monitoring of blazars and shows how observations in low states can be used to gain insight on the properties of the emission region.

Acknowledgements. I would like to thank Prof. A. Mastichiadis for helpful discussions and the anonymous referee for his/her comments that helped to improve the manuscript. Support for this work was provided by NASA through Einstein Postdoctoral Fellowship grant number PF3 140113 awarded by the Chandra 
X-ray Center, which is operated by the Smithsonian Astrophysical Observatory for NASA under contract NAS8-03060.

\section{Appendix A: Separation distance in the 2-SSC model}

In the 2-SSC model we treated the two components independently, i.e. we used as seed photons for the inverse Compton scattering only the synchrotron photons produced within each component. We calculate the minimum separation distance between the two components needed for this assumption to hold.

In what follows we use the following notation: single and double primes denote quantities measured in their respective comoving frames, while subscripts 1 and 2 refer to quantities of the first and second components.

The energy density of synchrotron photons of the two components as measured in their comoving frames is given by

$u_{\mathrm{syn}, 1}^{\prime}=\frac{\left.v_{\mathrm{p}} L_{\mathrm{syn}}^{\mathrm{obs}}\left(v_{\mathrm{p}}\right)\right|_{1}}{4 \pi c R_{1}^{2} \delta_{1}^{4}}$
$u_{\mathrm{syn}, 2}^{\prime \prime}=\frac{\left.v_{\mathrm{p}} L_{\mathrm{syn}}^{\mathrm{obs}}\left(v_{\mathrm{p}}\right)\right|_{2}}{4 \pi c R_{2}^{2} \delta_{2}^{4}}$,

where we approximated the total synchrotron luminosity by its value at the peak frequency $v_{\mathrm{p}}$ in each case. By looking at the SEDs in Fig. 7 one sees that the error introduced by this assumption is small. Taking also into account that $\left.v_{\mathrm{p}} L_{\mathrm{syn}}^{\mathrm{obs}}\left(v_{\mathrm{p}}\right)\right|_{1} /\left.v_{\mathrm{p}} L_{\mathrm{syn}}^{\mathrm{obs}}\left(v_{\mathrm{p}}\right)\right|_{2} \sim 0.6$ and using the values in Table 1 for the sizes and Doppler factors we find that

$\frac{u_{\mathrm{syn}, 1}^{\prime}}{u_{\mathrm{syn}, 2}^{\prime \prime}} \simeq 1.7 \times 10^{3}$.

Because of this large difference in the energy densities of synchrotron photons, one can pose the question: can the energy density of synchrotron photons from the first component as seen in the rest frame of the second one, be more important than the energy density of the internally produced?

To answer, one has to calculate the quantity $u_{\mathrm{syn}, 1}^{\prime \prime}$. We assume that the velocity vectors of the two components are parallel. Then their relative velocity and Lorentz factor are given by

$\beta_{\text {rel }}=\frac{\beta_{2}-\beta_{1}}{1-\beta_{1} \beta_{2}}$

$\Gamma_{\text {rel }}=\Gamma_{1} \Gamma_{2}\left(1-\beta_{1} \beta_{2}\right)$.

Assuming that $\Gamma_{1} \approx \delta_{1}=18$ and $\Gamma_{2} \approx \delta_{2}=34$ we find that $\beta_{\text {rel }}=0.56$ and $\Gamma_{\text {rel }}=1.2$.

Using the invariance of $u(\epsilon, \mu) / \epsilon^{3}$ and the transformation of the solid angle

$\mathrm{d} \Omega^{\prime \prime}=\frac{2 \pi}{\Gamma_{\text {rel }}^{2}\left(1-\beta_{\text {rel }} \mu^{\prime}\right)^{2}} \mathrm{~d} \mu^{\prime}$

we find that

$$
\begin{aligned}
u_{\mathrm{syn}, 1}^{\prime \prime} & =\int \mathrm{d} \epsilon^{\prime \prime} \int \mathrm{d} \mu^{\prime \prime} u_{\mathrm{syn}, 1}^{\prime \prime}\left(\epsilon^{\prime \prime}, \mu^{\prime \prime}\right) \\
& =\int \mathrm{d} \epsilon^{\prime} \int \mathrm{d} \mu^{\prime} \Gamma_{\mathrm{rel}}^{2}\left(1-\beta_{\mathrm{rel}} \mu^{\prime}\right)^{2} u_{\mathrm{syn}, 1}^{\prime}\left(\epsilon^{\prime}, \mu^{\prime}\right) \\
& =\frac{u_{\mathrm{syn}, 1}^{\prime}}{2} \Gamma_{\mathrm{rel}}^{2} \int_{\mu 12}^{1} \mathrm{~d} \mu^{\prime}\left(1-\beta_{\mathrm{rel}} \mu^{\prime}\right)^{2}
\end{aligned}
$$

where $\mu_{12}=r_{12} / \sqrt{r_{12}^{2}+R_{1}^{2}}$. In the above we assumed an isotropic synchrotron photon field in the comoving frame of the first component, i.e. $u_{\mathrm{syn}, 1}^{\prime}\left(\mu^{\prime}\right)=u_{\mathrm{syn}, 1}^{\prime} / 2$. The result of the integration is a function of $r_{12}$ and $\beta_{\text {rel }}$ :

$g\left(r_{12}, \beta_{\text {rel }}\right)=1-\mu_{12}-\beta_{\text {rel }}\left(1-\mu_{12}^{2}\right)+\frac{\beta_{\text {rel }}^{2}}{3}\left(1-\mu_{12}^{3}\right)$.

Combining the above, the condition $u_{\text {syn, } 1}^{\prime \prime} \lesssim u_{\text {syn, } 2}^{\prime \prime} / 3$ is written as

$g\left(r_{12}, \beta_{\text {rel }}\right)<\frac{2 u_{\mathrm{syn}, 2}^{\prime \prime}}{3 u_{\mathrm{syn}, 1}^{\prime} \Gamma_{\text {rel }}^{2}}$.

This results in $r_{12}>6 \times 10^{16} \mathrm{~cm}$. As long as the separation between the two is larger than the radius of the larger emitting region, it is safe to neglect the synchrotron photon field of the first component. For the opposite case, i.e. what is the contribution of the synchrotron photon field of the second component to the emission of the first one, a similar calculation is not necessary because the second component (i) moves away from the first one and (ii) it has a lower energy density $\left(u_{\text {syn, } 2}^{\prime \prime} \ll u_{\text {syn }, 1}^{\prime}\right)$.

\section{References}

Abdo, A. A., Ackermann, M., Ajello, M., et al. 2011, ApJ, 736, 131

Aharonian, F. A. 2000, New Astron., 5, 377

Aharonian, F., Akhperjanian, A. G., Anton, G., et al. 2009a, A\&A, 502, 749

Aharonian, F., Akhperjanian, A. G., Anton, G., et al. 2009b, ApJ, 696, L150

Atwood, W. B., Abdo, A. A., Ackermann, M., et al. 2009, ApJ, 697, 1071

Barres de Almeida, U., Ward, M. J., Dominici, T. P., et al. 2010, MNRAS, 408, 1778

Barres de Almeida, U., Tavecchio, F., \& Mankuzhiyil, N. 2014, MNRAS, 441, 2885

Bloom, S. D., \& Marscher, A. P. 1996, ApJ, 461, 657

Boettcher, M. 2012, Fermi \& Jansky Proceedings - eConf C1111101 [arXiv: 1205.0539]

Böttcher, M., \& Dermer, C. D. 1998, ApJ, 501, L51

Böttcher, M., Reimer, A., Sweeney, K., \& Prakash, A. 2013, ApJ, 768, 54

Burrows, D. N., Hill, J. E., Nousek, J. A., et al. 2005, Space Sci. Rev., 120, 165

Cao, G., \& Wang, J. 2014, ApJ, 783, 108

Cerruti, M., Zech, A., Boisson, C., \& Inoue, S. 2012, in AIP Conf. Ser. 1505, eds. F. A. Aharonian, W. Hofmann, \& F. M. Rieger, 635

Coppi, P. S., \& Aharonian, F. A. 1999, ApJ, 521, L33

Dermer, C. D., \& Humi, M. 2001, ApJ, 556, 479

Dermer, C. D., \& Schlickeiser, R. 1993, ApJ, 416, 458

Dermer, C. D., Schlickeiser, R., \& Mastichiadis, A. 1992, A\&A, 256, L27

Dimitrakoudis, S., Mastichiadis, A., Protheroe, R. J., \& Reimer, A. 2012, A\&A, 546, A 120

Dimitrakoudis, S., Petropoulou, M., \& Mastichiadis, A. 2014, Astropart. Phys., 54,61

Donnarumma, I., Vittorini, V., Vercellone, S., et al. 2009, ApJ, 691, L13

Finke, J. D., Razzaque, S., \& Dermer, C. D. 2010, ApJ, 712, 238

Fossati, G., Maraschi, L., Celotti, A., Comastri, A., \& Ghisellini, G. 1998, MNRAS, 299, 433

Fossati, G., Buckley, J. H., Bond, I. H., et al. 2008, ApJ, 677, 906

Ghisellini, G., \& Madau, P. 1996, MNRAS, 280, 67

Hauser, M., Möllenhoff, C., Pühlhofer, G., et al. 2004, Astron. Nachr., 325, 659

H.E.S.S. Collaboration, Abramowski, A., Acero, F., et al. 2012, A\&A, 539, A149

Hinton, J. A., \& Hofmann, W. 2009, ARA\&A, 47, 523

Holder, J. 2012, Astropart. Phys., 39, 61

Jahoda, K., Swank, J. H., Giles, A. B., et al. 1996, in EUV, X-Ray, and GammaRay Instrumentation for Astronomy VII, eds. O. H. Siegmund, \& M. A. Gummin, SPIE Conf. Ser., 2808, 59

Katarzyński, K., Ghisellini, G., Tavecchio, F., et al. 2005, A\&A, 433, 479

Konopelko, A., Mastichiadis, A., Kirk, J., de Jager, O. C., \& Stecker, F. W. 2003, ApJ, 597, 851

Krawczynski, H., Coppi, P. S., \& Aharonian, F. 2002, MNRAS, 336, 721

Krawczynski, H., Hughes, S. B., Horan, D., et al. 2004, ApJ, 601, 151

Lefa, E., Rieger, F. M., \& Aharonian, F. 2011, ApJ, 740, 64

Mannheim, K., \& Biermann, P. L. 1992, A\&A, 253, L21

Maraschi, L., Ghisellini, G., \& Celotti, A. 1992, ApJ, 397, L5 
M. Petropoulou: Time dependent modelling of PKS 2155-304 in a low state

Massaro, E., Tramacere, A., Perri, M., Giommi, P., \& Tosti, G. 2006, A\&A, 448, 861

Mastichiadis, A., \& Kirk, J. G. 1995, A\&A, 295, 613

Mastichiadis, A., \& Kirk, J. G. 1997, A\&A, 320, 19

Mastichiadis, A., \& Moraitis, K. 2008, A\&A, 491, L37

Mastichiadis, A., Protheroe, R. J., \& Kirk, J. G. 2005, A\&A, 433, 765

Mastichiadis, A., Petropoulou, M., \& Dimitrakoudis, S. 2013, MNRAS, 434, 2684

Moraitis, K., \& Mastichiadis, A. 2011, A\&A, 525, A40

Mücke, A., Protheroe, R. J., Engel, R., Rachen, J. P., \& Stanev, T. 2003, Astropart. Phys., 18, 593

Nalewajko, K., Begelman, M. C., \& Sikora, M. 2014, ApJ, 789, 161

Petropoulou, M., \& Mastichiadis, A. 2011, A\&A, 532, A11

Petropoulou, M., \& Mastichiadis, A. 2012a, MNRAS, 426, 462
Petropoulou, M., \& Mastichiadis, A. 2012b, MNRAS, 421, 2325

Petropoulou, M., \& Mastichiadis, A. 2013, in Eur. Phys. J. Web Conf., 61, 5013 Petropoulou, M., Giannios, D., \& Dimitrakoudis, S. 2014, MNRAS, submitted [arXiv: 1405.2091$]$

Reimer, A. 2012, J. Phys. Conf. Ser., 355, 012011

Sahu, S., Oliveros, A. F. O., \& Sanabria, J. C. 2013, Phys. Rev. D, 87, 103015

Sikora, M., Begelman, M. C., \& Rees, M. J. 1994, ApJ, 421, 153

Sikora, M., Błażejowski, M., Begelman, M. C., \& Moderski, R. 2001, ApJ, 554, 1

Tammi, J., \& Duffy, P. 2009, MNRAS, 393, 1063

Tavecchio, F., Maraschi, L., \& Ghisellini, G. 1998, ApJ, 509, 608

Ulrich, M.-H., Maraschi, L., \& Urry, C. M. 1997, ARA\&A, 35, 445

Wagner, R. 2008, in Blazar Variability across the Electromagnetic Spectrum, PoS(BLAZARS2008)013 